\title{
Genomic Characterization of a Multidrug-Resistant and Hypermucoviscous/Hypervirulent Klebsiella quasipneumoniae subsp. similipneumoniae ST4417 Isolated from a Sewage Treatment Plant
}

\author{
João Pedro Rueda Furlan,, Inara Fernanda Lage Gallo,' \\ Tatiana Amabile de Campos, ${ }^{2}$ and Eliana Guedes Stehling ${ }^{1}$
}

\begin{abstract}
Aim: Clinical strains of Klebsiella quasipneumoniae subsp. similipneumoniae have been reported worldwide. Multidrug-resistant (MDR) hypermucoviscous (hm)/hypervirulent (hv) lineages have become a global problem for public health worldwide. Therefore, this study aimed to characterize by whole-genome sequencing a MDR$\mathrm{hm} / \mathrm{hv}$ K. quasipneumoniae subsp. similipneumoniae SWT10 strain belonging to the new sequence type ST4417 isolated from a sewage treatment plant.

Results: The SWT10 strain was recovered from a sewage treatment plant in Brazil and presented the hm and MDR phenotypes. Resistome analysis showed antimicrobial resistance genes associated with resistance to fluoroquinolones, $\beta$-lactams, tetracyclines, trimethoprim, aminoglycosides, sulfonamides, macrolides, and fosfomycin as well as several heavy metal resistance genes. Virulome analysis showed virulence factors related to hv lineages. Multilocus sequence typing analysis revealed the new ST4417, which was grouped in CC1584 by the minimum-spanning tree. Besides, five plasmid incompatibility groups, two prophage-related sequences, and 66 genomic islands were detected.

Conclusion: This study reports for the first time the genome sequence of a MDR-hm/hv K. quasipneumoniae subsp. similipneumoniae recovered from the environment, which contributes to a better understanding about these lineages as well as for surveillance studies worldwide.
\end{abstract}

Keywords: Klebsiella quasipneumoniae subsp. similipneumoniae, multidrug-resistant, hypermucoviscous, hypervirulent, water

\section{Introduction}

$\boldsymbol{K}$ LEBSIELLA QUASIPNEUMONIAE SUBSP. Similipneumoniae, also termed as belonging to the phylogenetic group KpIIb of Klebsiella pneumoniae, has been reported worldwide, mainly in clinical sources. Hypermucoviscous (hm)/ hypervirulent (hv) lineages are closely related to invasive infections, which confer resistance to intracellular killing and phagocytosis. ${ }^{1}$ Aquatic environments have been described as reservoir of multidrug-resistant (MDR) bacteria, which contribute to the spread of MDR pathogens. ${ }^{2}$ The emergence of MDR pathogens in several ecological spheres, including the environment, became a global threat to public health. Therefore, this study aimed to characterize an MDR$\mathrm{hm} / \mathrm{hv} K$. quasipneumoniae subsp. similipneumoniae strain isolated from a sewage treatment plant.

\section{Materials and Methods}

In 2016, a $K$. quasipneumoniae subsp. similipneumoniae strain (SWT10) was recovered from a sewage treatment plant in Brasília, Distrito Federal, Brazil. The hm phenotype was determined by string test (presence of a viscous filament $\geq 5 \mathrm{~mm}$ after stretching using a bacteriological loop). ${ }^{3}$ The antimicrobial resistance profile was determined by the disk diffusion method and minimum inhibitory concentration (MIC) following the guidelines of the Clinical and Laboratory Standards Institute (CLSI; M100, 28th ed.) and the Brazilian Committee on Antimicrobial Susceptibility Testing (BrCast; version 9.0, 2019).

The pattern of resistance was determined according to Magiorakos et al. ${ }^{4}$ Metal tolerance profile was performed using a range of $0.005-256 \mathrm{mM}$ of copper, silver, arsenic,

\footnotetext{
${ }^{1}$ Departamento de Análises Clínicas, Toxicológicas e Bromatológicas, Faculdade de Ciências Farmacêuticas de Ribeirão Preto, Universidade de São Paulo, Ribeirão Preto, São Paulo, Brazil.

${ }^{2}$ Departamento de Biologia Celular, Instituto de Ciências Biológicas, Universidade de Brasília, Brasília, Brazil.
} 
Table 1. Genome Analysis of Multidrug-Resistant and Hypermucoviscous/Hypervirulent KLEBSIELLA Quasipneumoniae SubsP. Similipneumoniae SWT10 Isolated from a SeWage Treatment Plant

\begin{tabular}{|c|c|}
\hline Characteristics & SWT10 \\
\hline Sequence type & 4417 \\
\hline Clonal complex & 1584 \\
\hline$w z i$ allele & 290 \\
\hline \multicolumn{2}{|l|}{ Antimicrobial resistance genes to } \\
\hline$\beta$-lactams & bla $a_{\mathrm{OKP}-\mathrm{B}-5}$ \\
\hline (Fluoro) quinolones & $\operatorname{oq} x A, \operatorname{oq} x B$ \\
\hline Tetracyclines & tetA \\
\hline Aminoglycosides & $\operatorname{aadA} 2$ \\
\hline Sulfonamides & sull \\
\hline Trimethoprim & dfrA32 \\
\hline Macrolides & ereA \\
\hline Fosfomycin & fos $A$ \\
\hline Efflux systems and regulators & acrA, acrR, marA, marR, sox, soxS, ramA, rob, sdiA, fis \\
\hline \multicolumn{2}{|l|}{ Heavy metal resistance genes to } \\
\hline Copper & pcoABCDERS, copAD, nlpE \\
\hline Silver & silCERS \\
\hline Arsenic & $\operatorname{arsCH}$ \\
\hline Chromium & $\operatorname{chrA}$ \\
\hline Tellurium & teh $A B$, terABCDEFXZ \\
\hline Nickel & $n i k A B C D E$ \\
\hline Nickel/cobalt & $\operatorname{rcn} A B$ \\
\hline Copper/silver & cusA \\
\hline Magnesium/cobalt & $m g t A$ \\
\hline Cadmium/zinc/cobalt & $c z c A$ \\
\hline Chromium/tellurium/selenium & $\operatorname{ruvB}$ \\
\hline Magnesium/cobalt/nickel/manganese & $\operatorname{corA}$ \\
\hline Quaternary ammonium compounds & sugE \\
\hline \multicolumn{2}{|l|}{ Virulence genes to } \\
\hline Type 1 fimbriae & fimABCDEFGHIK \\
\hline Type 3 fimbriae & $m r k A B C D F$ \\
\hline Enterobactin & entB \\
\hline Aerobactin & iutA \\
\hline Salmochelin & iroBCN \\
\hline Colibactin & $c l b B L$ \\
\hline Ferric enterobactin & fepCG \\
\hline Ferric iron uptake & $k f u A B C$ \\
\hline Ferric uptake regulator & fur \\
\hline Pullulanase secretion & pulBCDEFGHIJKLMNOS \\
\hline Escherichia coli common pilus & ecpABCDER \\
\hline Allantoin metabolism & allBR \\
\hline Mammalian cell entry & mceIG \\
\hline Plasmids & IncFIB(Mar), IncHI1B, IncR, Col(IMGS31), Col440I \\
\hline Prophage-related sequences $(n)$ & 2 \\
\hline Genomic islands $(n)$ & 66 \\
\hline GenBank accession number & VOIK01000000 \\
\hline
\end{tabular}

magnesium, cobalt, nickel, manganese, cadmium, zinc, chromium, tellurium, and selenium. ${ }^{5}$

The genomic DNA was extracted using the PureLink ${ }^{\mathrm{TM}}$ Genomic DNA Mini Kit (Thermo Fisher Scientific) and the whole genome sequencing was performed on an Illumina MiSeq platform (Illumina, Inc.), using $250 \mathrm{bp}$ paired-end reads. De novo genome assembly was accomplished using CLC Genomics Workbench 12 (Qiagen, Denmark) and the contigs were annotated using the NCBI Prokaryotic Genome Annotation Pipeline (PGAP) v.3.2. ${ }^{6}$ Antimicrobial resistance genes, plasmid replicons, and sequence type (ST) were analyzed using ResFinder 3.2, PlasmidFinder 2.0, and MLST 2.0, respectively. ${ }^{7-9}$ Detection of mutations in the lipid A-Ara4N pathway (ArnA DH/FT, ArnB, ArnC, ArnT,
PmrL, PmrJ, and PhoB) and lipid A (LpxM, YciM, MrgB, PagP, PhoP, PhoQ, PmrA, PmrB, EptA, and EptB) that confer resistance to polymyxin $\mathrm{B}$ and colistin, respectively, was researched using the $K$. quasipneumoniae subsp. similipneumoniae ATCC 700603 as reference. ${ }^{10}$

The clonal complex (CC) was determined using the PHYLOViZ 2.0 software based on seven housekeeping genes (multilocus sequence typing [MLST]) of 4488 STs described so far (September 2019). ${ }^{11}$ The whole-genome single nucleotide polymorphism (wgSNP) analysis was performed using the CSI Phylogeny $1.4^{12}$ with the $K$. quasipneumoniae subsp. similipneumoniae ATCC 700603 as reference. ${ }^{10}$ Virulence genes, efflux systems, and regulators and capsular type were determined using the $K$. pneumoniae 


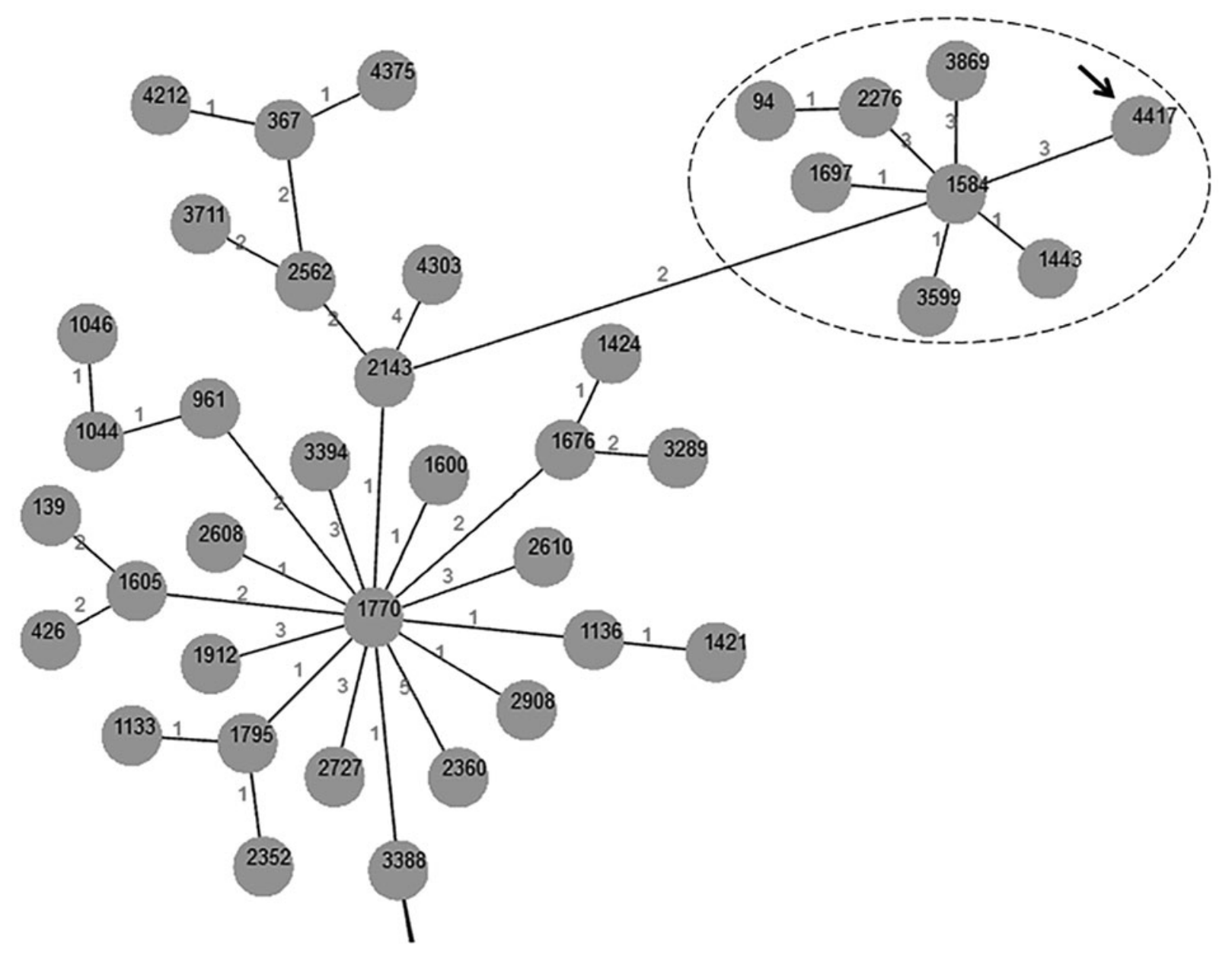

FIG. 1. Minimum-spanning tree based on multilocus sequence typing of 4488 STs using the PHYLOViZ software. The CC1584 was highlighted by a dashed circle. The ST4417 was highlighted by an arrow. The numbers between circles represent the number of allelic mismatches between STs. ST, sequence type.

database. ${ }^{13}$ Antibacterial biocide and metal resistance genes were detected using the BacMet database. ${ }^{14}$ Phage-related sequences and genomic islands were detected using the PHASTER $^{15}$ and IslandViewer 4 plus SIGI-HMM, ${ }^{16}$ respectively.

\section{Results and Discussion}

The SWT10 strain exhibited an hm phenotype (positive string test). It presented an MDR profile to cefazolin, cefuroxime, cefaclor, ceftriaxone, cefotaxime, ceftazidime, nalidixic acid, levofloxacin, ciprofloxacin, norfloxacin, lomefloxacin, ofloxacin, tetracycline, doxycycline, streptomycin, trimethoprim/sulfamethoxazole, sulfonamides, and trimethoprim and MIC $0.5 \mathrm{mg} / \mathrm{L}$ for polymyxin B and $1 \mathrm{mg} / \mathrm{L}$ for colistin, being classified as susceptible. This strain showed different MICs for metals, including copper $(5 \mathrm{mM})$, silver $(0.005 \mathrm{mM})$, arsenic $(45 \mathrm{mM})$, magnesium $(>100 \mathrm{mM})$, cobalt $(1 \mathrm{mM})$, nickel $(1 \mathrm{mM})$, manganese $(4 \mathrm{mM})$, zinc $(5 \mathrm{mM})$, cadmium (0.6 mM), chromium (2 mM), tellurium (62.5 mM), and selenium $(6.25 \mathrm{mM})$.

A total of $1,279.668(2 \times 250 \mathrm{bp})$ paired-end reads were generated with $58 \times$ coverage. In total, 5,789 protein-coding sequences, 247 pseudogenes, 76 tRNAs, 10 ncRNAs, and 8
rRNAs were identified, with guanine-cytosine (GC) content of $57 \%$. Resistome analysis showed antimicrobial resistance genes for $\beta$-lactams ( bla $_{\text {OKP-B-5 }}$ ), fluoroquinolones (oqxA and oq $x B$ ), tetracyclines (tetA), aminoglycosides (aadA2), sulfonamides (sul1), trimethoprim (dfrA32), macrolides (ereA), and fosfomycin (fosA). Mutations in ArnA DH/FT (T431N), ArnB (A244T), LpxM (A26T), PagP (S6A), and EptA (L11V; A154V) were detected; however, the SWT10 strain was susceptible for polymyxin B and colistin.

Five plasmid incompatibility groups were detected, including IncFIB(Mar), IncHI1B, IncR, Col(IMGS31), and Col440I. In addition, the efflux systems and regulator genes (acrA, acrR, marA, marR, soxR, soxS, ramA, rob, sdiA, and fis), as well as resistance genes to copper ( $p c o A B C D E R S$, copAD, and $n l p E$ ), silver (silCERS), copper/silver (cusA), nickel/cobalt ( $r c n A B)$, arsenic (ars $C H)$, magnesium/ cobalt/nickel/manganese (corA), nickel (nikABCDE), magnesium/cobalt (mgtA), cadmium/zinc/cobalt ( $c z c A)$, chromium (chrA), tellurium (tehAB and terABCDEFXZ), chromium/tellurium/selenium $(r u v B)$, and quaternary ammonium compounds ( $\operatorname{sugE}$ ), were also detected (Table 1).

The SWT10 strain was assigned to capsular type wzi290KL47. Several virulence factors were detected, such as iutA (aerobactin), entB (enterobactin), iroBCN (salmochelin), $c l b B L$ 
(colibactin), fepCG (ferric enterobactin), fimABCDEFGHIK (type 1 fimbriae), $m r k A B C D F$ (type 3 fimbriae), ecpABCDER (Escherichia coli common pilus), $k f u A B C$ (ferric iron uptake), allBR (allantoin metabolism), mceIG (mammalian cell entry), pulBCDEFGHIJKLMNOS (pullulanase secretion), and fur (ferric uptake regulator). In addition, two intact phage-related sequences (Salmonella phage SEN5 [24.8 kb] and Haemophilus phage $\mathrm{SuMu}[37.5 \mathrm{~kb}]$ ) and 66 genomic islands related to the genome evolution and microbial adaptability that ranged in size from 4,258 to $6,5905 \mathrm{bp}$ (average $11,960 \pm 10,590 \mathrm{bp}$ ) were detected (Table 1).

MLST analysis revealed the new allele for $p h o E$ gene (allele 453). The association of this new allele with other alleles belonging to the six other housekeeping genes ( $\mathrm{gapA}$ [18], infB [22], mdh [18], pgi [16], rpoB [20], and tonB [79]) generated the new ST4417. The minimum-spanning tree showed that ST4417 grouped in CC1584 differing by a 3-loci variant (Fig. 1). The wgSNP distance from SWT10 strain to K. quasipneumoniae subsp. similipneumoniae ATCC 700603 was 0.30298. Clinical strains of K. pneumoniae belonging to ST1584 (CC1584) carrying extended-spectrum $\beta$-lactamase (ESBL) and Klebsiella pneumoniae carbapenemase (KPC) as well as virulence genes have already been reported on different continents, including in South America. ${ }^{17,18}$

The STW10 strain presented the positive string test and the virulence genes that are used for the classification of hm/hv lineage. ${ }^{19}$ OKP-B-type chromosomal $\beta$-lactamases are closely related to $K$. quasipneumoniae, and OKP-B-5-producing $K$. quasipneumoniae has been commonly reported in human clinical sources, such as urine and wound. ${ }^{20}$ Liver abscess and bloodstream caused by hm/hv K. quasipneumoniae subsp. quasipneumoniae were reported in North America and Europe. ${ }^{21,22}$ Besides, MDR ESBL-, MCR-1-, and MCR-8.2-producing $K$. quasipneumoniae have been described in animals (e.g., dog, chicken, pig, and turtle) from China, Japan, and Taiwan. ${ }^{23-26}$

In Brazil, an isolate of KPC-2- and OKP-B-6-producing $K$. quasipneumoniae subsp. similipneumoniae was reported in nosocomial infection $^{27}$ and the co-occurrence of CTX-M-15 and KPC-9 in a clinical strain of MDR $K$. quasipneumoniae subsp. similipneumoniae was described in India. ${ }^{28}$ The characteristics of $\mathrm{hm} / \mathrm{hv}$ in MDR and non-MDR K. quasipneumoniae subsp. similipneumoniae have already been reported; however, not in the same strain, as described in this study (i.e., MDR-hm/ hv K. quasipneumoniae subsp. similipneumoniae $)^{22,29}$

\section{Conclusion}

The association of antimicrobial resistance and virulence determinants represents a problem for therapy since these determinants favor the likelihood of colonization as well as the persistence of these pathogens in both patients and the environment. In summary, we report the first genome sequence of an MDR-hm/hv K. quasipneumoniae subsp. similipneumoniae recovered from the environment. This genome sequence contributes to a better understanding of MDR-hm/hv $K$. quasipneumoniae subsp. similipneumoniae and provides relevant data to be used in surveillance studies.

\section{Acknowledgments}

The authors thank the team of curators of the Institut Pasteur MLST and whole genome MLST databases for curating the data and making them publicly available.

\section{Availability of Data}

This Whole Genome Shotgun project has been deposited at DDBJ/ENA/GenBank under the accession number VOIK00000000. The version described in this article is VOIK00000000.

\section{Disclosure Statement}

No competing financial interests exist.

\section{Funding Information}

The authors thank the Fundação de Amparo à Pesquisa do Estado de São Paulo (FAPESP) (Grant No. 2018/01890-3) and the Coordenação de Aperfeiçoamento de Pessoal de Nível Superior (CAPES) (Grant No. 88882.180855/2018-01 and code 001) for fellowships. This study was supported by Fundação de Amparo à Pesquisa do Estado de São Paulo (FAPESP) (Grant No. 2018/19539-0).

\section{References}

1. Brisse, S., V. Passet, and P.A. Grimont. 2014. Description of Klebsiella quasipneumoniae sp. nov., isolated from human infections, with two subspecies, Klebsiella quasipneumoniae subsp. quasipneumoniae subsp. nov. and Klebsiella quasipneumoniae subsp. similipneumoniae subsp. nov., and demonstration that Klebsiella singaporensis is a junior heterotypic synonym of Klebsiella variicola. Int. J. Syst. Evol. Microbiol. 64:3146-3152.

2. Khan, F.A., B. Hellmark, R. Ehricht, B. Söderquist, and J. Jass. 2018. Related carbapenemase-producing Klebsiella isolates detected in both a hospital and associated aquatic environment in Sweden. Eur. J. Clin. Microbiol. Infect. Dis. 37:2241-2251.

3. Shon, A.S., Bajwa, R.P.S., and T.A. Russo. 2013. Hypervirulent (hypermuscoviscous) Klebsiella pneumoniae: a new and dangerous breed. Virulence 4:107-113.

4. Magiorakos, A.P., A. Srinivasan, R.B. Carey, Y. Carmeli, M.E. Falagas, C.G. Giske, S. Harbarth, J.F. Hindler, G. Kahlmeter, B. Olsson-Liljequist, D.L. Paterson, L.B. Rice, J. Stelling, M.J. Struelens, A. Vatopoulos, J.T. Weber, and D.L. Monnet. 2012. Multidrug-resistant, extensively drugresistant and pandrug-resistant bacteria: an international expert proposal for interim standard definitions for acquired resistance. Clin. Microbiol. Infect. 18:268-281.

5. Deredjian, A., C. Colinon, E. Brothier, S. Favre-Bonté, B. Cournoyer, and S. Nazaret. 2011. Antibiotic and metal resistance among hospital and outdoor strains of Pseudomonas aeruginosa. Res. Microbiol. 162:689-700.

6. Tatusova, T., M. DiCuccio, A. Badretdin, V. Chetvernin, E.P. Nawrocki, L. Zaslavsky, A. Lomsadze, K.D. Pruitt, M. Borodovsky, and J. Ostell. 2016. NCBI prokaryotic genome annotation pipeline. Nucleic Acids Res. 44:66146624.

7. Zankari, E., H. Hasman, S. Cosentino, M. Vestergaard, S. Rasmussen, O. Lund, F.M. Aarestrup, and M.V. Larsen. 2012. Identification of acquired antimicrobial resistance genes. J. Antimicrob. Chemother. 67:2640-2644.

8. Larsen, M.V., S. Cosentino, S. Rasmussen, C. Friis, H. Hasman, R.L. Marvig, L. Jelsbak, T. Sicheritz-Pontén, D.W. Ussery, F.M. Aarestrup, and O. Lund. 2012. Multilocus sequence typing of total genome sequenced bacteria. J. Clin. Micobiol. 50:1355-1361. 
9. Carattoli, A., E. Zankari, A. García-Fernández, M. Voldby Larsen, O. Lund, L. Villa, F. Møller Aarestrup, and H. Hasman. 2014. In silico detection and typing of plasmids using PlasmidFinder and plasmid multilocus sequence typing. Antimicrob. Agents Chemother. 58:3895-3903.

10. Elliott, A.G., D. Ganesamoorthy, L. Coin, M.A. Cooper, and M.D. Cao. 2016. Complete genome sequence of Klebsiella quasipneumoniae subsp. similipneumoniae strain ATCC 700603. Genome Announc. 4:e00438-16.

11. Francisco, A.P., C. Vaz, P.T. Monteiro, J. Melo-Cristino, M. Ramirez, and J.A. Carriço. 2012. PHYLOViZ: phylogenetic inference and data visualization for sequence based typing methods. BMC Bioinformatics 13:87.

12. Kaas, R.S., P. Leekitcharoenphon, F.M. Aarestrup, and O. Lund. 2014. Solving the problem of comparing whole bacterial genomes across different sequencing platforms. PLoS One 9:e104984.

13. Jolley, K.A., J.E. Bray, and M.C.J. Maiden. 2018. Openaccess bacterial population genomics: BIGSdb software, the PubMLST.org website and their applications. Wellcome Open Res. 3:124.

14. Pal, C., J. Bengtsson-Palme, C. Rensing, E. Kristiansson, and D.G. Larsson. 2014. BacMet: antibacterial biocide and metal resistance genes database. Nucleic Acids Res. 42: D737-D743.

15. Arndt, D., J. Grant, A. Marcu, T. Sajed, A. Pon, Y. Liang, D.S. Wishart. 2016. PHASTER: a better, faster version of the PHAST phage search tool. Nucleic Acids Res. 44:W16-W21.

16. Bertelli, C., Laird, M. R., Williams, K. P., Simon Fraser University Research Computing Group, B.Y. Lau, G. Hoad, G.L. Winsor, and F.S.L. Brinkman. 2017. IslandViewer 4: expanded prediction of genomic islands for larger-scale datasets. Nucleic Acids Res. 45:W30-W35.

17. Yan, J.J., P.X. Zheng, M.C. Wang, S.H. Tsai, L.R. Wang, and J.J. Wu. 2015. Allocation of Klebsiella pneumoniae bloodstream isolates into four distinct groups by ompK36 typing in a Taiwanese University Hospital. J. Clin. Microbiol. 53:3256-3263.

18. Rojas, L.J., G.M. Weinstock, E. De La Cadena, L. Diaz, R. Rios, B.M. Hanson, J.S. Brown, P. Vats, D.S. Phillips, H. Nguyen, K.M. Hujer, A. Correa, M.D. Adams, F. Perez, E. Sodergren, A. Narechania, P.J. Planet, M.V. Villegas, R.A. Bonomo, and C.A. Arias. 2017. An analysis of the epidemic of Klebsiella pneumoniae carbapenemase-producing $K$. pneumoniae: convergence of two evolutionary mechanisms creates the "perfect storm". J. Infect. Dis. 217:82-92.

19. Catalán-Nájera, J.C., U. Garza-Ramos, and H. BarriosCamacho. 2017. Hypervirulence and hypermucoviscosity: two different but complementary Klebsiella spp. phenotypes? Virulence 8:1111-1123.

20. Long, S.W., S.E. Linson, M. Ojeda Saavedra, C. Cantu, J.J. Davis, T. Brettin, and R.J. Olsen. 2017. Whole-genome sequencing of human clinical Klebsiella pneumoniae isolates reveals misidentification and misunderstandings of Klebsiella pneumoniae, Klebsiella variicola, and Klebsiella quasipneumoniae. mSphere 2:e0290-17.

21. Breurec, S., B. Melot, B. Hoen, V. Passet, K. Schepers, S. Bastian, and S. Brisse. 2016. Liver abscess caused by infection with community-acquired Klebsiella quasipneumoniae subsp. quasipneumoniae. Emerg. Infect. Dis. 22:529.
22. Arena, F., L. Henrici De Angelis, F. Pieralli, V. Di Pilato, T. Giani, F. Torricelli, M.M. D'Andrea, and G.M. Rossolini. 2015. Draft genome sequence of the first hypermucoviscous Klebsiella quasipneumoniae subsp. quasipneumoniae isolate from a bloodstream infection. Genome Announc. 3:e00952-15.

23. Li, C.F., H.L. Tang, C.S. Chiou, K.C. Tung, M.C. Lu, and Y.C. Lai. 2018. Draft genome sequence of CTX-M-type $\beta$ lactamase-producing Klebsiella quasipneumoniae subsp. similipneumoniae isolated from a Box turtle. J. Glob. Antimicrob. Resist. 12:235-236.

24. Yang, X., L. Liu, Z. Wang, L. Bai, and R. Li. 2019. Emergence of mcr-8.2-bearing Klebsiella quasipneumoniae of animal origin. J. Antimicrob. Chemother. 74:2814-2817.

25. Li, X.P., R.Y. Sun, J.Q. Song, L.X. Fang, R.M. Zhang, X.L. Lian, X.P. Liao, Y.H. Liu, J. Lin, and J. Sun. 2019. Withinhost heterogeneity and flexibility of $\mathrm{mcr}-1$ transmission in chicken gut. Int. J. Antimicrob. Agents S0924-S8579: 30253-30255.

26. Harada, K., T. Shimizu, Y. Mukai, K. Kuwajima, T. Sato, M. Usui, Y. Tamura, Y. Kimura, T. Miyamoto, Y. Tsuyuki, A. Ohki, and Y. Kataoka. 2016. Phenotypic and molecular characterization of antimicrobial resistance in Klebsiella spp. isolates from companion animals in Japan: clonal dissemination of multidrug-resistant extended-spectrum $\beta$ lactamase-producing Klebsiella pneumoniae. Front. Microbiol. 7:1021.

27. Nicolás, M.F., P.I.P. Ramos, F. Marques de Carvalho, D.R.A. Camargo, C. de Fátima Morais Alves, G. Loss de Morais, L.G.P. Almeida, R.C. Souza, L.P. Ciapina, A.C.P. Vicente, R.S. Coimbra, and A.T. Ribeiro de Vasconcelos. 2018. Comparative genomic analysis of a clinical isolate of Klebsiella quasipneumoniae subsp. similipneumoniae, a KPC-2 and OKP-B-6 beta-lactamases producer harboring two drug-resistance plasmids from Southeast Brazil. Front. Microbiol. 169:220.

28. Shankar, C., S. Karunasree, A. Manesh, and B. Veeraraghavan. 2019. First report of whole-genome sequence of colistin-resistant Klebsiella quasipneumoniae subsp. similipneumoniae producing KPC-9 in India. Microb. Drug Resist. 25:489-493.

29. Ozer, E.A., A.R. Morris, F. Krapp, C.S. Henry, K.E. Tyo, W.W. Lathem, and A.R. Hauser. 2016. Draft genome sequence of a multidrug-resistant Klebsiella quasipneumoniae subsp. similipneumoniae isolate from a clinical source. Genome Announc. 4:e00422-16.

Address correspondence to: Eliana Guedes Stehling, PhD Departamento de Análises Clínicas, Toxicológicas e Bromatológicas Faculdade de Ciências Farmacêuticas de Ribeirão Preto Universidade de São Paulo Avenida do Café S/N. Monte Alegre Ribeirão Preto 14040-903 São Paulo Brazil

E-mail: elianags@usp.br 